\title{
Beyond Folklore: Observations on Fragmented Traffic
}

\author{
Colleen Shannon, David Moore, and K. C. Claffy
}

\begin{abstract}
Fragmented IP traffic is a poorly understood component of the overall mix of traffic on the Internet. Many assertions about the nature and extent of fragmented traffic are anecdotal rather than empirical. In this paper we examine the causes and attributes of measured fragment traffic, in particular, the effects of NFS, streaming media, networked video games, tunneled traffic, and the prevalence of packet fragmentation due to improperly configured machines.

To understand the prevalence, causes, and effects of fragmented IP traffic, we have collected and analyzed seven multiday traces from four sources. These sources include a university commodity access link, two highly aggregated commercial exchange points, and a local NAP. Although there is no practical method of ascertaining whether any data provide a representative sample of all Internet traffic, we include data sources that cover several different types of WANs with traffic from commercial entities, educational and research institutions, and large government facilities.

The dominant causes of fragmentation are streaming media and tunneled traffic. Although rumored to be the main impetus for IP packet fragmentation, NFS is not among the top ten causes.
\end{abstract}

Index Terms-Fragment, fragmentation, measurement, TCP/IP, traffic measurement.

\section{INTRODUCTION}

$\mathbf{T}$ HE Internet Protocol (IP) was designed to facilitate communication between heterogenous networks. It serves as a least-common-denominator protocol that allows computers differing in architectures, operating systems, and applications, connected by varying routes, paths, and protocols, to exchange information. IP must be able to handle differences in maximum sizes of transmitted packets on dissimilar networks. While it is trivial to move packets from a network with a smaller maximum transmission unit (MTU) to a network with a larger MTU, the reverse is challenging. To overcome this obstacle, the IPv4 protocol performs fragmentation: a router breaks the datagram up into smaller individual pieces called fragments. Each fragment has its own IP header, which is a replica of the original datagram header. Thus each fragment has the same identification, protocol, source IP address, and destination IP address as the original IP packet. To distinguish fragments and allow correct reassembly, the offset field of each fragment contains the distance, measured in 8-byte units, between the beginning of the original datagram and the beginning of that particular fragment. The first fragment by definition has its offset set to 0 , the second

Manuscript received December 12, 2001; revised July 7, 2002; approved by IEEE/ACM TRANSACTIONS ON NETWORKING Editor J. Rexford. This work was supported by DARPA under NGI Contract N66001-98-2-8922, the National Science Foundation under Grant NCR-9711092, and CAIDA members.

The authors are with CAIDA, San Diego Supercomputer Center, University of California at San Diego, La Jolla, CA 92003 USA (e-mail cshannon@caida.org; dmoore@caida.org; kc@caida.org).

Digital Object Identifier 10.1109/TNET.2002.805028 fragment has as its offset value the payload size of the first fragment, and so on. All of the fragments except the last have the "more fragments" bit set so that the destination host waits to receive all of the fragments before reassembling them into the original IP datagram. The size of each fragment usually corresponds to the size of the MTU of the subsequent link minus the length of the header that is added to each fragment. After disassembly of the original datagram, fragments are sent out into the network and are routed independently toward their destination. By providing an automatic intranetwork mechanism for handling disparate MTU sizes, IP allows end hosts to exchange traffic with no explicit knowledge about the path between them.

In their 1987 paper [1], Kent and Mogul established that packet fragmentation is a suboptimal method of handling packets as they traverse a network. Although current technology mitigates some of the described problems with consumption of bandwidth, packet switching, and CPU resources, the overall argument that fragmentation is detrimental remains valid. The adverse effects of fragmentation on network performance and infrastructure continue to negatively impact wide area transport. First, an intermediate router must perform the fragmentation. The router must process the fragment with its main CPU, rather than utilizing the specialized hardware in a line card, commonly called the "fast path." This CPU-intensive operation may impair the ability of the fragmenting router to efficiently process non-fast-path traffic. The additional fragmented packets increase the load on all routers and networks between the initial router and the end host. Finally, once the fragments reach their destination, they must be reassembled by the end host. The loss of any fragment causes the destination host to drop the entire packet. This in turn forces the source host to repeat transmission of a datagram that will likely be fragmented once again. Researchers have shown that in certain specific, controlled circumstances fragmentation can improve performance [2]; however, those observations do not apply to backbone links. Despite widespread advances in the intervening thirteen years, IP packet fragmentation is still "considered harmful."

Since the work of Kent and Mogul, many untested hypotheses about the causes and effects of fragmented IP traffic have come to be treated as fact. Foremost is the assertion that fragmented traffic no longer exists. Others in the networking community accept the existence of fragmented traffic on LANs, but believe its scope does not extend to backbone links. Further common beliefs include that only UDP traffic is fragmented, that NFS is the source of all fragmented packet traffic, that fragmented IP traffic on the whole is decreasing, and that certain misconfigurations are causing an increase in fragmented traffic. These beliefs as a group are not tenable, since several are mutually exclusive (e.g., the overall volume of fragmented traffic cannot be simultaneously increasing and decreasing). While one recent publication 


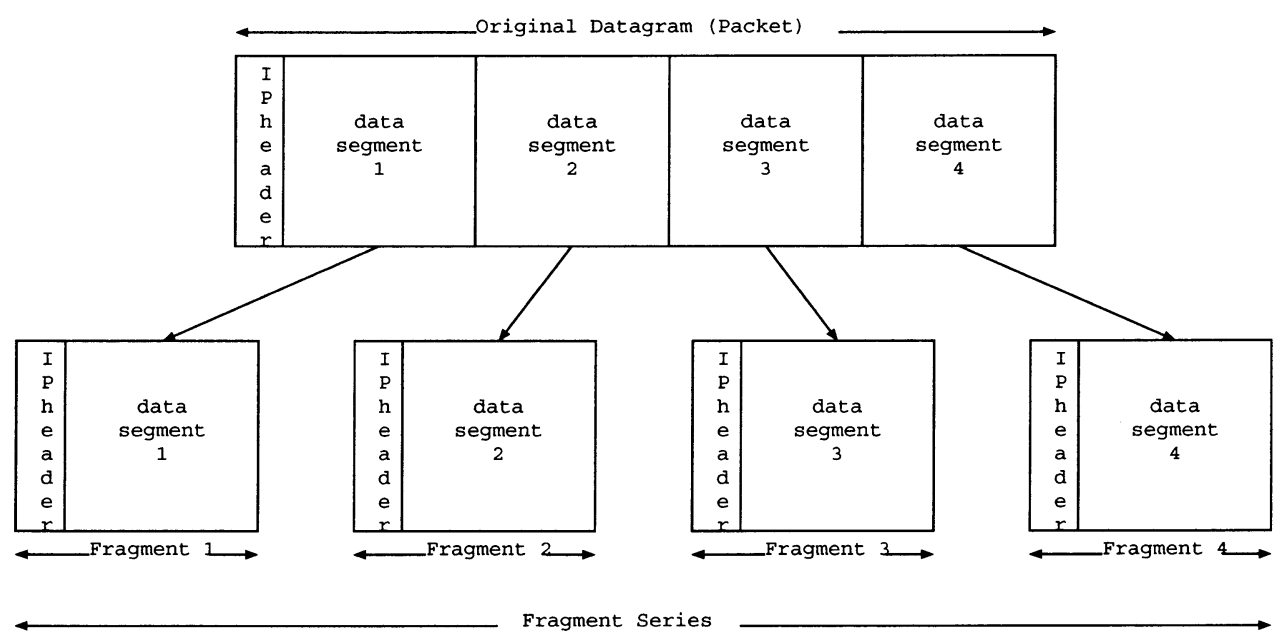

Fig. 1. Composition of a fragment series.

suggests that IP packet fragmentation is increasing [3], all other fragment folklore has no basis in current network measurement.

IP packet fragmentation continues to play a small but vital role in facilitating communication between hosts on the Internet. The proliferation of protocols that send packets with different MTUs necessitates a system flexible enough to accommodate these variations. IP packet fragmentation increases the robustness and efficacy of IP as a universal protocol. In this paper, we examine the character and effects of fragmented IP traffic as monitored on highly aggregated Internet links.

The paper is organized as follows. Section II defines terminology we use to describe fragmented traffic. Sources of data and our methodologies for analysis are presented in Section III. In Section IV, we present our results characterizing fragmented traffic. Finally, Section V summarizes the current effects of fragmented traffic on the monitored links.

\section{TERMINOLOGY}

This section introduces the terminology used in our discussion of IP packet fragmentation. Several of these terms are illustrated in Fig. 1.

As described in RFC 1191 [4], the Path MTU is the smallest MTU of all of the links on a path from a source host to a destination host. In the context of this paper, values observed for a Path MTU reflect the smallest MTU of all links between the source and the passive monitor.

We define an original datagram as an IP datagram that will be fragmented because its size exceeds the MTU of the next link on its path to its destination. Packet fragment, or simply fragment, refers to a packet containing a portion of the payload of an original datagram. While for the purposes of this paper, the terms packet and datagram are synonymous, we will use original datagram and packet fragment in the interest of clarity. A fragment series, or simply series, is the ordered list (as monitored on the network) of fragments derived from a single original datagram.

The size of the series will be used to refer to the total number of bytes in the series, while the length of the series describes the number of fragments in the series. A data segment is a portion of the original packet payload that becomes the payload of a single fragment.

The first fragment is the packet containing the original IP header and the first data segment of the payload of the original datagram. The last fragment is the packet containing the last portion of the payload of the original datagram. Because fragments can be transmitted in any order and because packets can be reordered as they pass through a network, the first observed and last observed fragments do not necessarily contain the first and last segments of the payload of the original datagram (respectively), and are thus not necessarily the first or last fragment of the series.

The first fragment is frequently equal in size to the largest fragment in each series. The largest fragment size is greater than or equal to the size of the other fragments in the series.

Similarly, the last fragment is not always the smallest fragment in a series. So the smallest fragment size is less than or equal to the other fragment sizes in a series. RFC 791 [5] does not specify how fragments must be sized, other than that the payload of all of the non-last fragments must be a multiple of 8 bytes in length.

Because the IP protocol permits networks to drop, duplicate, or reorder packets, the individual fragment packets for a single original datagram may not arrive at the destination in transmission order. We define a series as complete when the fragmented packets monitored provide sufficient coverage of the original data segment to allow reconstruction of the transmitted datagram (i.e., reordering or duplication may have occurred, but no fragments are missing). Conversely, an incomplete series (Fig. 2) does not have sufficient information to reconstruct the original datagram; some part of the payload never reached our monitor.

A series is in-order (Fig. 3) if the fragments are observed arriving sequentially; i.e., we never monitor a fragment with an offset lower than its predecessors. Conversely, a series is considered to be in reverse-order (Fig. 4) if its fragments have offsets that never increase. A computer producing in-order series transmits data segment 1 through data segment $N$, while a computer producing reverse-order series transmits data segment $N$ 


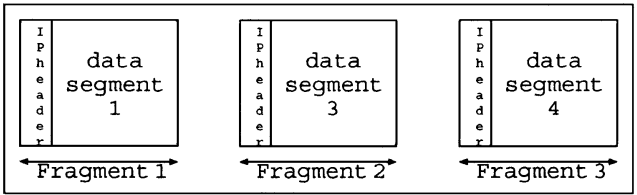

Fig. 2. Example of an incomplete series.

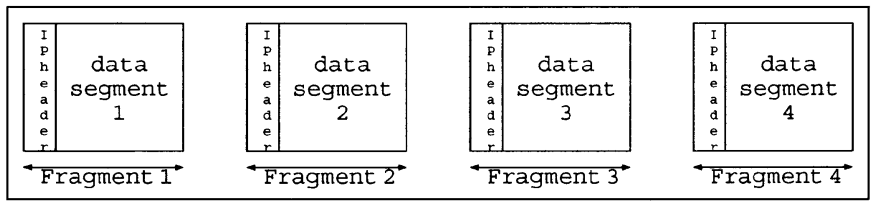

Fig. 3. Example of an in-order series.

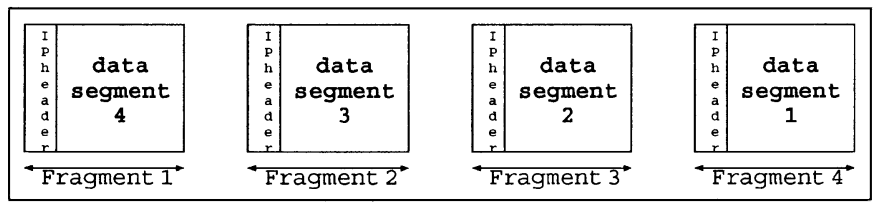

Fig. 4. Example of a reverse-order series.

through data segment 1 . Only one fragment needs to be delivered out of order for us to observe a reverse-order two-fragment series. However, we cannot necessarily correlate the order in which we received the packet fragments and the order in which they were transmitted by the fragmenting router, since the fragments can be reordered by the network. For longer series, it is less probable that an exact reversal of the fragment order occurs in the network than it is that the ordering is due to reverse-order transmission.

A series contains a duplicate (Fig. 5) if at least two of its fragments cover the exact same portion of the original payload.

An overlapping series (Fig. 6) has at least two fragment packets that contain overlapping portions of the original payload when the two fragments are not duplicates. Conversely, a nonoverlapping series has no overlapping fragments. Note that the "teardrop" denial of service attack [6], [7] sends large fragments that are overlapping except for a single byte, thereby exhausting buffer resources in certain fragment reassembly implementations.

We define a correct series (Fig. 7) as a series that is complete, with no overlapping or duplicated fragments. Any order of fragment arrival is acceptable in a correct series.

\section{MethodOLOGY}

\section{Measurement Sites}

Data sets for this study were collected from three different locations, summarized in Table I. The first data source for this study was a link at MAE-west. We used an Apptel Point OC12 card [8] to collect traffic exchanged by customers that peer at MAE-west. No intra-customer traffic is observed at this location. Traffic across SDNAP, a regional exchange point located in San Diego, CA, was the second data source for this paper. We used libpcap [9] and an off-the-shelf 100 Mbit ethernet card to monitor this traffic. Using a FORE ATM OC3 card [10], we

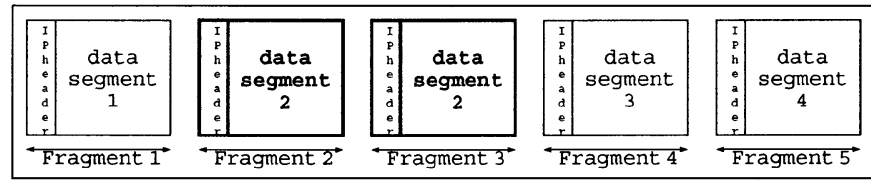

Fig. 5. Example of a duplicate series.

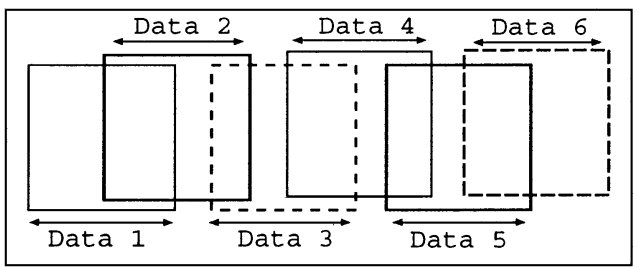

Fig. 6. Example of an overlapping series.

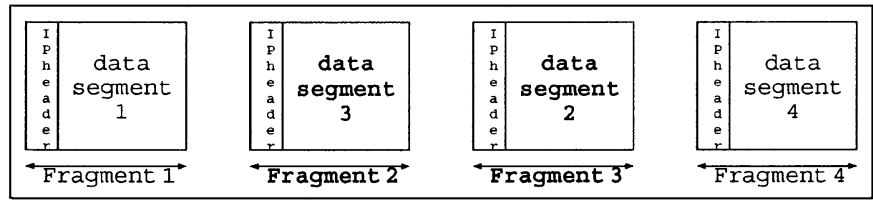

Fig. 7. Example of a correct series. Note that this series is not in-order.

monitored the commodity access link that connects the University of California, San Diego campus (including such entities as the San Diego Supercomputer Center and the Scripps Institute of Oceanography) to CERFnet. At our final location, traffic was collected from a link between Ames Internet Exchange (AIX) and MAE-west, using a WAND DAG OC3 card [11].

The numbers of unique source hosts for each data set (shown in Table I) were limited to hosts that sent at least three packets over the lifetime of the trace. This filtering was applied to provide a more accurate count of the actual number of hosts transmitting across the link, since the MAE-west data sets contained at least one random source denial-of-service attack.

\section{Traffic Monitoring}

Due to the high volume of traffic at some of the measurement sites, a specialized tool, crl_frag_capture, collected the data for this study. crl_frag_capture relies on the CoralReef [12] software suite for header capture, interval handling, and data aggregation. We gleaned only packet headers; we attempted no analysis of the payload portion of each packet. We organized the data we collected into hour-long intervals for postprocessing. We collected four types of data:

frags.pcap - a full header trace in libpcap [9] format containing only fragmented traffic packets (either offset $>0$ or "more fragments" bit set).

$s r c \_i p . t 2$ - an aggregated table of nonfragmented traffic containing the number of packets and bytes seen per source IP address.

proto_ports_folded.t2 - an aggregated table of nonfragmented traffic with the number of packets and bytes seen per 3-tuple of IP protocol, source port, and destination port. Since a significant amount of monitored traffic travels between a well-known port and an ephemeral port, additional aggregation was done for commonly occurring ports to keep the tuple table 
TABLE I

DATASETS USED IN A STUDY—MARCH 2001

\begin{tabular}{l|r|r|r|r|r}
\hline \multicolumn{1}{c|}{ Dataset } & \multicolumn{2}{|c|}{ Length } & \multicolumn{3}{c}{ Characteristics } \\
\hline & Start Time (UTC) & Duration (hours) & Packets (kpkts) & Bytes (MB) & Src Hosts* \\
\hline \hline CERF-IN & Fri Mar 9 02:01 & 252.00 & $2,797,266$ & $1,439,570$ & $2,745,493$ \\
CERF-OUT & Fri Mar 9 02:01 & 252.00 & $3,394,283$ & $1,559,170$ & 37,242 \\
SDNAP & Fri Mar 9 01:36 & 259.58 & $1,073,321$ & 646,677 & 328,094 \\
MAEWEST-1 & Fri Mar 9 01:35 & 75.00 & $5,307,429$ & $2,203,614$ & $1,277,423$ \\
MAEWEST-2 & Tue Mar 13 02:12 & 132.00 & $8,991,449$ & $3,963,302$ & $1,691,880$ \\
AIX-1 & Fri Mar 9 01:38 & 58.00 & $8,781,881$ & $3,281,324$ & $2,684,104$ \\
AIX-2 & Mon Mar 12 04:35 & 49.00 & $8,070,586$ & $3,743,040$ & $2,478,624$ \\
\hline
\end{tabular}

*Unique IP source addresses that sent at least three packets over the trace lifetime.

size from exceeding memory space on the machine. A list of 19 ports $^{1}$ was chosen from preliminary studies of nonfragmented traffic on these links. For each packet with a source or destination matching one of these common ports, the ephemeral port is set to 0 , causing all traffic for each of these 19 services to fall into only 19 entries in our tuple table. This method maintains the port that matches the traffic to a specific application, while discarding the dynamically generated, meaningless port. Additionally, all ports above 32767 were bucketed as 32768 , since the ports in this range are typically dynamically allocated and we found in preliminary studies that no well known ports above 32767 had a significant volume of traffic.

length.t2 - a table of nonfragmented traffic aggregated by the number of packets and bytes seen with each IP packet size.

The collection of full header traces for nonfragmented traffic was not feasible due to the high volume of traffic on the monitored links. Furthermore, partitioning of the data into independent tables for source IP address, protocol/ports, and packet length obscures the original relationships between these fields.

\section{Fragment Processing}

For an in-depth analysis of IP packet fragmentation, constituent fragments from each original datagram were assembled into a fragment series. Fragments were separated into discrete series using the identification, protocol, source IP address and destination IP address fields, since those fields uniquely define fragments of an original datagram. The payload of the original packet was not reconstructed, since the offset and size of each fragment are sufficient to infer the basic properties of fragmented traffic.

The grouping of fragments into series is sensitive to the chosen reassembly timeout. On the one hand, we wish to provide sufficient time for all fragments in each series to be monitored even with significant network delays. However, we also need to account for the possibility of a wraparound of the IP ID field. While we would not ordinarily expect the IP ID field to wrap in a short period of time, there are a few cases in which we do monitor duplicate IP ID fields from a single host in a short period of time. For example, we observed tunnel ingress points that generated sufficient traffic to wrap their IP ID fields in only a few minutes. If the timeout is too lengthy, the likelihood of incorrectly assigning fragments from disparate

\footnotetext{
${ }^{1}$ The specific ports in aggregation application order are: 80, 53, 25, 443, $27015,110,113,37,20,119,5000,6112,6667,6688,6699,6970,8888,9000$, 27005 .
}

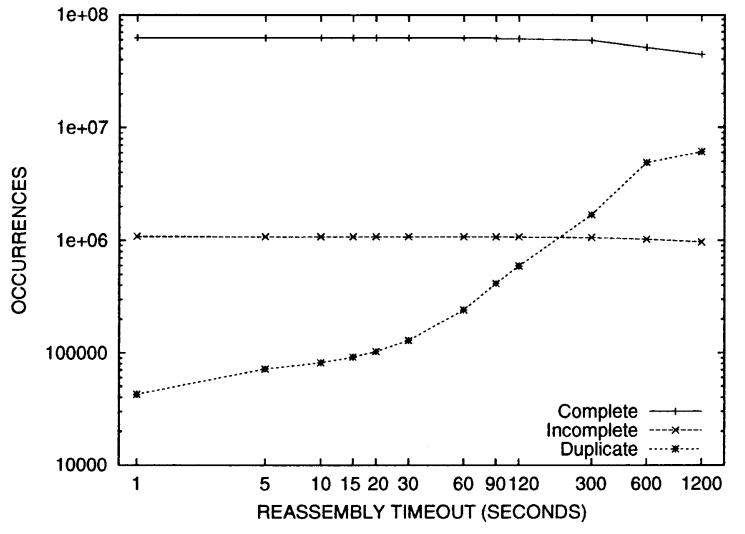

Fig. 8. The effects of various reassembly timeouts on complete, incomplete, and duplicate fragment series.

original datagrams into the same fragments series increases. As shown in Fig. 8, the number of duplicate fragments in each series (which can be an indicator of IP ID wraparound) increases across tested fragment series timeouts. Conversely, the number of incomplete series decreases with increasing timeout magnitude. Because there exists no point at which erroneous duplicates are minimized while complete series are maximized, we have chosen to use a timeout of $15 \mathrm{~s}$, as it is the maximum advisable delay before reassembly recommended in RFC 791 [5].

\section{Application Mapping}

To discern which applications and services produce the most fragmented traffic, we map the protocol, source port, and destination port fields of each IP packet header to a named application by choosing the first matching rule from an ordered collection of protocol/port patterns. For this study, we used CAIDA's passive monitor report generator application list. ${ }^{2}$ The list contained 92 application to port mappings, including common wellknown ports from the IANA port assignment list [13], as well as emerging multimedia, file sharing, and video game applications [such as RealAudio, Quake, Napster, eDonkey2000, and FastTrack (KaZaA)]. For example, traffic to and from ports 80 and 8080 are classified as WWW traffic, while connections to port 21 are classified as FTP data. Because passive FTP utilizes dynamically allocated ports, we cannot distinguish it using well-known ports. As described in Section IV-D, we were able

${ }^{2}$ The mapping code and application/port list used in this study (from CoralReef 3.5.2) as well as the current CAIDA list can be obtained from the authors or by emailing coral-info@caida.org. 


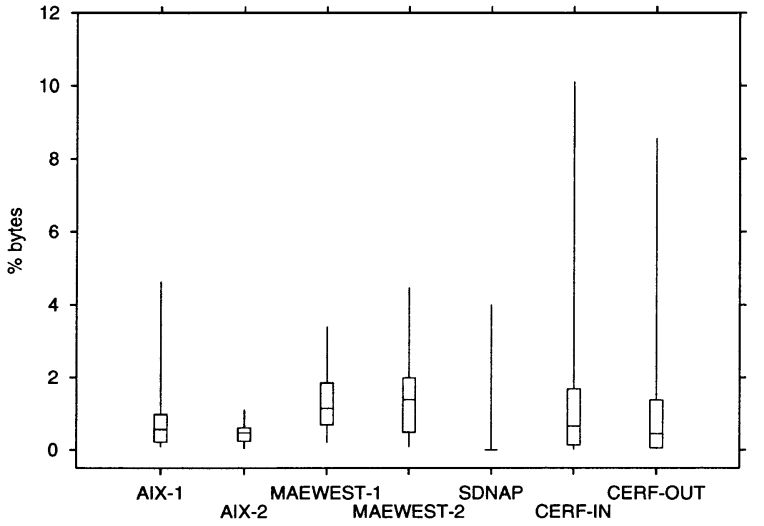

(a)

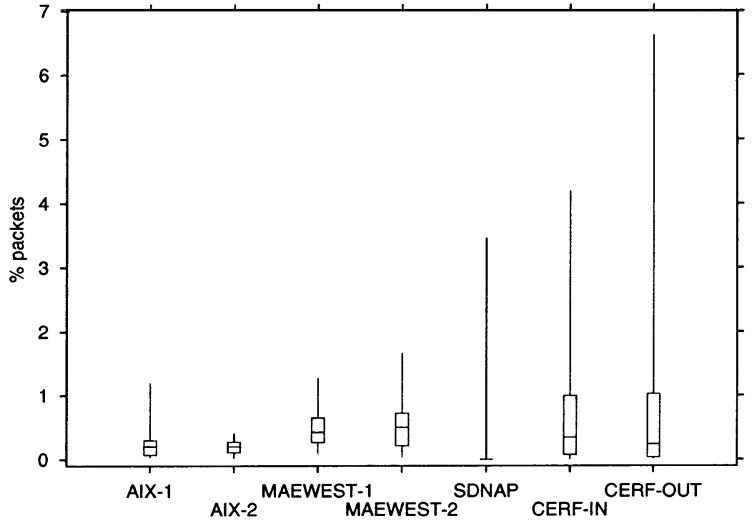

(b)

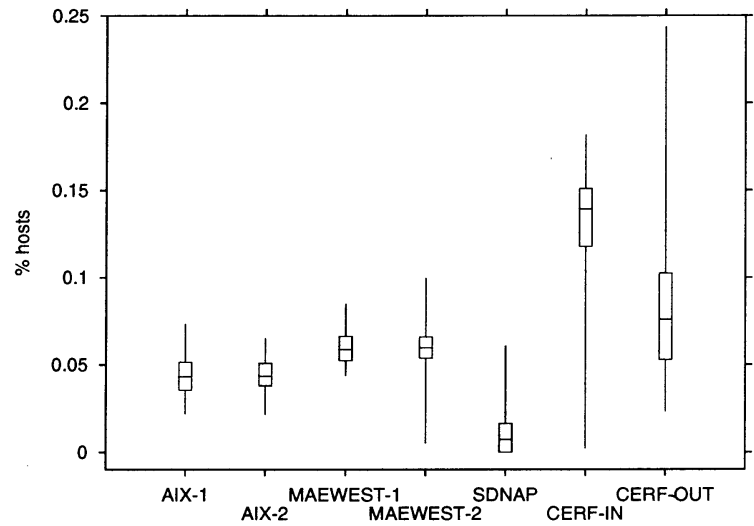

(c)

Fig. 9. Percentage of fragmented traffic by: (a) bandwidth; (b) packets; or (c) unique hosts for 1-h intervals for each trace. The candlestick lines show minimum and maximum percentage of traffic seen in an hour, the bottom and top of the box show the 25th and 75th percentiles, and the line inside the box shows the median value.

TABLE II

PREVALENCE OF FRAGMENTED AND NONFRAGMENTED IP TRAFFIC

\begin{tabular}{c||r|r|r||r|r|r}
\hline \multicolumn{1}{c||}{ Trace } & \multicolumn{3}{c||}{ Fragmented } & \multicolumn{3}{c}{ Non-Fragmented } \\
\hline & Pkts(\%) & Bytes $(\%)$ & \multicolumn{1}{c}{ Hosts $^{1}(\%)$} & Pkts $(\%)$ & Bytes(\%) & Hosts $^{1}(\%)$ \\
\hline \hline CERF-IN & 0.675 & 1.556 & 0.042 & 99.325 & 98.444 & 99.989 \\
CERF-OUT & 0.742 & 1.283 & 0.177 & 99.258 & 98.717 & 100.000 \\
SDNAP & 0.069 & 0.090 & 0.023 & 99.931 & 99.910 & 99.998 \\
MAEWEST-1 & 0.534 & 1.459 & 0.174 & 99.466 & 98.541 & 99.994 \\
MAEWEST-2 & 0.578 & 1.573 & 0.183 & 99.422 & 98.427 & 99.996 \\
AIX-1 & 0.269 & 0.835 & 0.172 & 99.731 & 99.165 & 99.973 \\
AIX-2 & 0.250 & 0.590 & 0.162 & 99.750 & 99.410 & 99.974 \\
\hline
\end{tabular}

to use control traffic to identify almost all applications using ephemeral ports.

\section{RESULTS}

\section{A. Overall Trends in Fragmented Traffic}

Table II shows the percentage of fragmented and nonfragmented traffic found in each data set. We observed hosts sending both fragmented traffic and nonfragmented traffic, so the host percentages may total more than $100 \%$. Although the overall volume of fragmented traffic was small, it was also highly variable. Fig. 9 shows the variance in the number of fragmented packets, number of bytes carried in fragmented packets, and number of hosts sending fragmented traffic.

The nonfragmented traffic measured by both the AIX and MAE-west monitors demonstrated diurnal cycles. The traffic at
SDNAP does not share the strongly cyclical nature of traffic at the other two locations, although it does show a daily decrease in traffic late at night (Pacific Standard Time).

Fig. 10 shows time series plots of the nonfragmented traffic. Note that Fig.10(e) and (f) do not exclude random source Denial of Service attacks. These attacks produce spikes in the number of hosts generating traffic with no periodic temporal patterns [14].

\section{B. Classification of Fragmented Traffic}

Fragment series can be categorized by the order in which the monitor received their constituent packets. Table III shows the breakdown of all series based on the following attributes (as defined in Section II): correct, complete, in-order, reverse-order, overlapping, and duplicate. Of all series, $98.3 \%$ are complete, meaning that they contain sufficient information to reconstruct the original datagram. Correct series (Fig. 7) account for $98.2 \%$ of all series. Of complete series, $90.4 \%$ are in-order (Fig. 3) and $7.9 \%$ are reverse-order (Fig. 4). Of all complete series, $0.1 \%$ are either overlapping (Fig. 6) or duplicate (Fig. 5) series; both are attributes that impede exact determination of ordering. Of all complete series, $0.002 \%$ have overlapping fragments and $0.15 \%$ contain duplicates.

Of all monitored series, $1.6 \%$ are correct series that were neither in-order nor reverse-order; they were likely reordered in transit. In May 2000, Paxson et al. [15] observed that approximately $0.3 \%$ of all packets arrive out of order. Thus it appears 


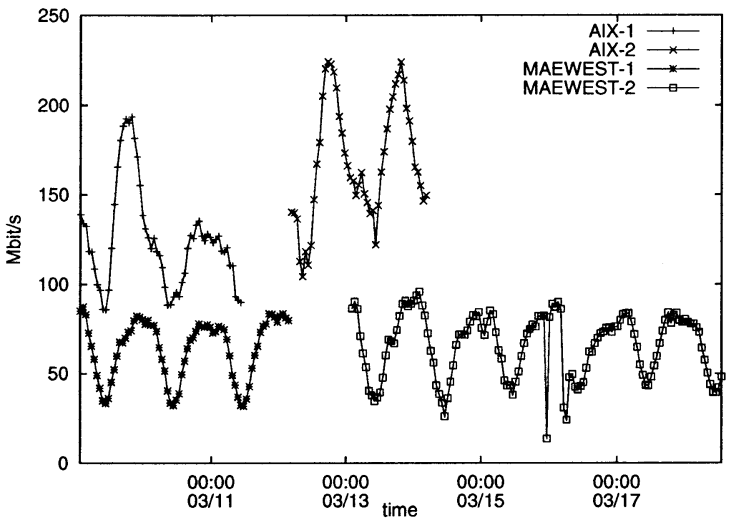

(a)

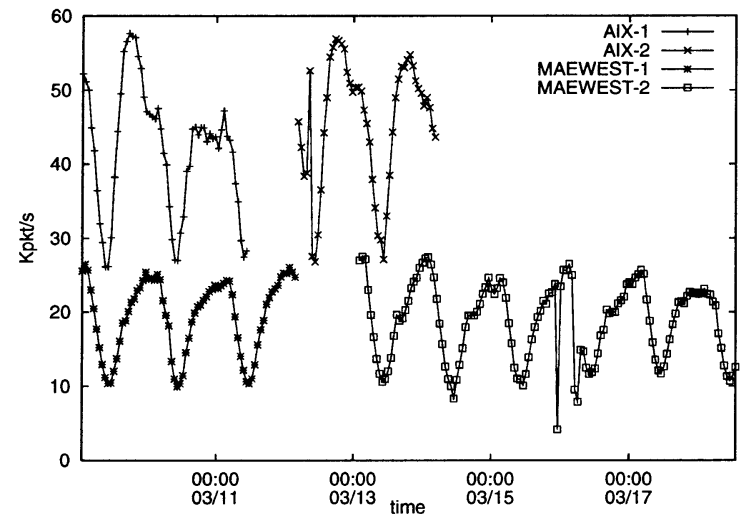

(c)

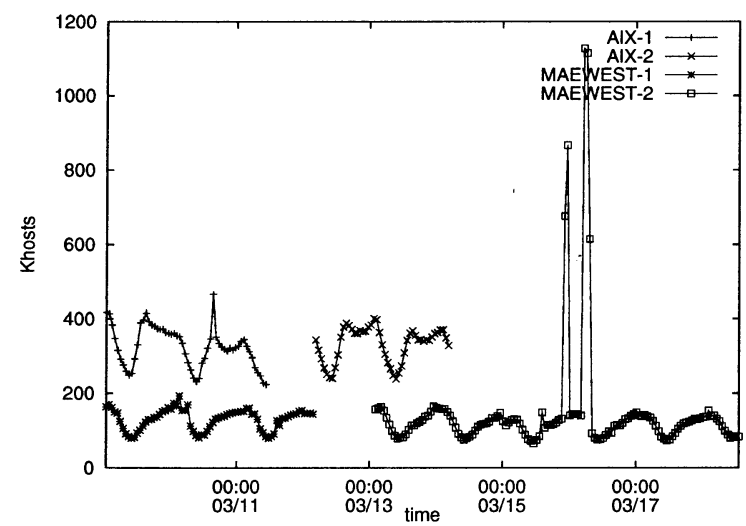

(e)

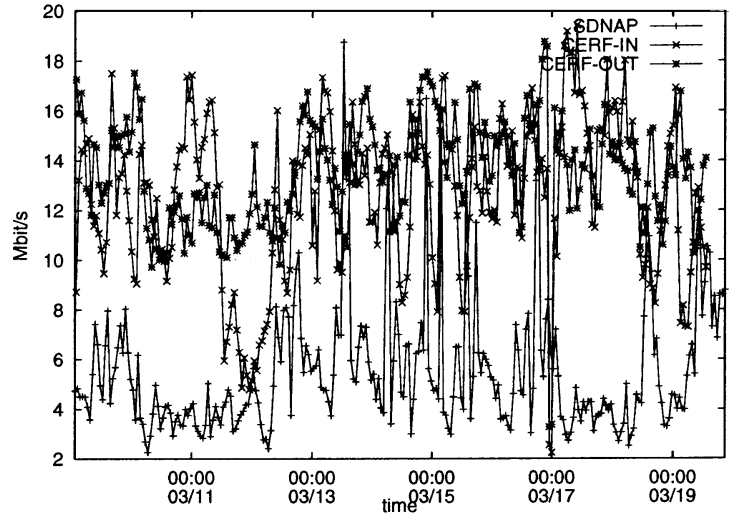

(b)

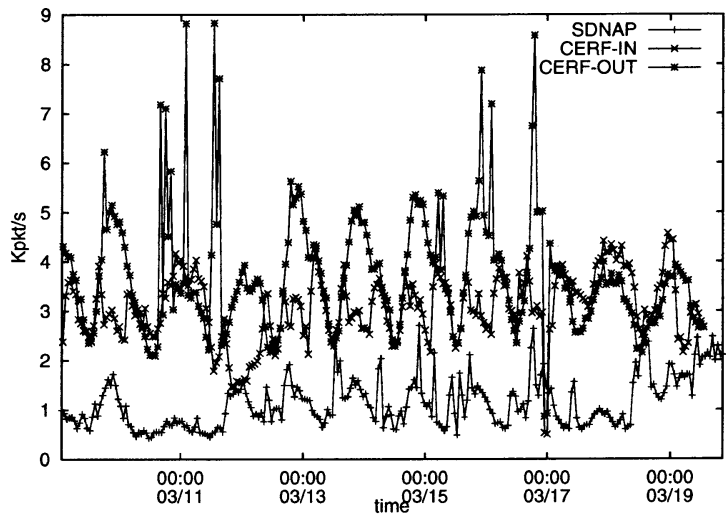

(d)

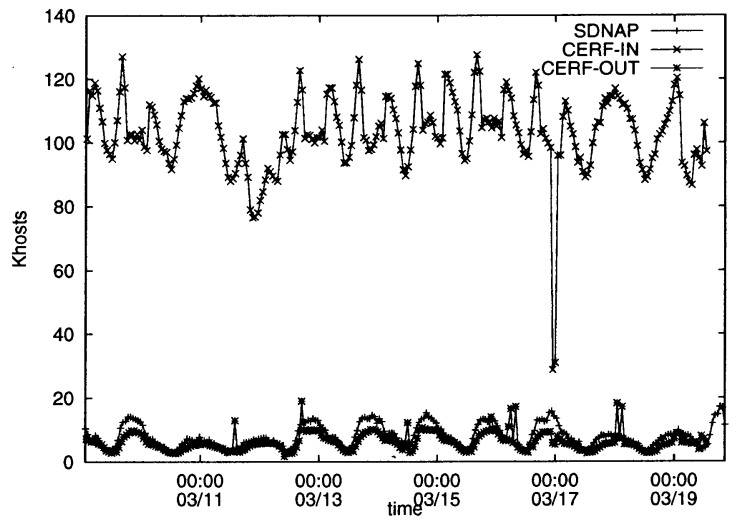

(f)

Fig. 10. (a), (b) Average hourly bandwidth. (c), (d) Packets. (e), (f) Unique hosts for nonfragmented traffic.

TABLE III

TOP SERIES KINDS FROM ALL SERIES

\begin{tabular}{c|c|c|c|c|c|r|r}
\hline \multicolumn{7}{c|}{ Category } & \multicolumn{2}{c}{ Occurrence } \\
\hline Correct & Complete & In-Order & Reverse & Overlap & Duplicate & \# Series & $\%$ Series \\
\hline \hline YES & YES & YES & - & - & - & $56,411,943$ & 89. \\
YES & YES & - & YES & - & - & $4,936,210$ & 7.8 \\
YES & YES & - & - & - & - & $1,028,327$ & 1.6 \\
- & - & YES & YES & - & - & 653,167 & 1.0 \\
- & - & YES & - & - & - & 339,362 & 0.53 \\
- & YES & - & - & - & YES & 89,288 & 0.14 \\
- & - & - & YES & - & - & 69,627 & 0.11 \\
- & - & - & - & - & - & 17,626 & 0.028 \\
- & - & YES & YES & - & YES & 990 & 0.0016 \\
- & YES & - & - & YES & YES & 974 & 0.0015 \\
\hline
\end{tabular}

that fragmented traffic has a greater probability of being reordered by the network than nonfragmented traffic. However, we have no way to quantify the overall frequency of out-of-order nonfragmented packets in our data sets so we cannot test this hypothesis.

Reverse-order series are not problematic; in fact, they can actually be beneficial since a host receiving a reverse-order series can use the fragment length and offset fields of the first received packet to immediately allocate correctly sized buffers, rather than growing or chaining buffers as subsequent fragments arrive.

\section{Characteristics of Fragment Traffic}

To clearly portray the characteristics of fragmented traffic, we use graphs generated from data collected at the Ames Internet Exchange because they demonstrate the basic properties 


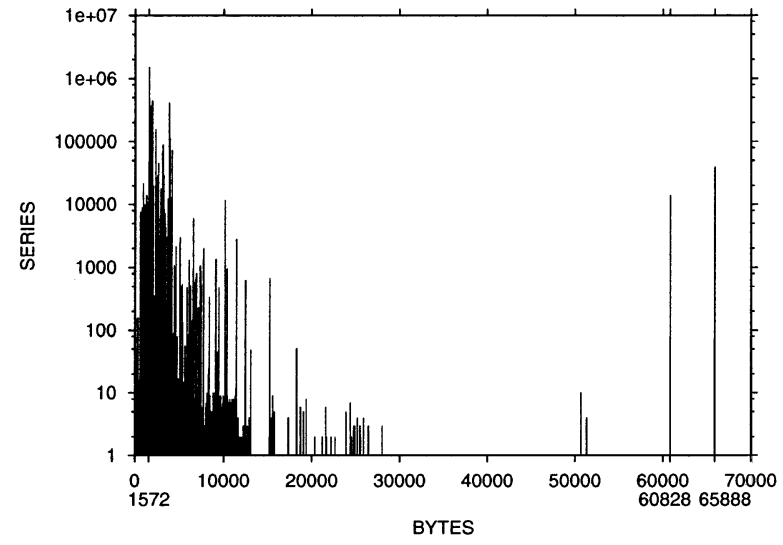

Fig. 11. Number of bytes transmitted per correct series for trace AIX-2. Note that this includes the bytes in all of the IP headers for each fragment.

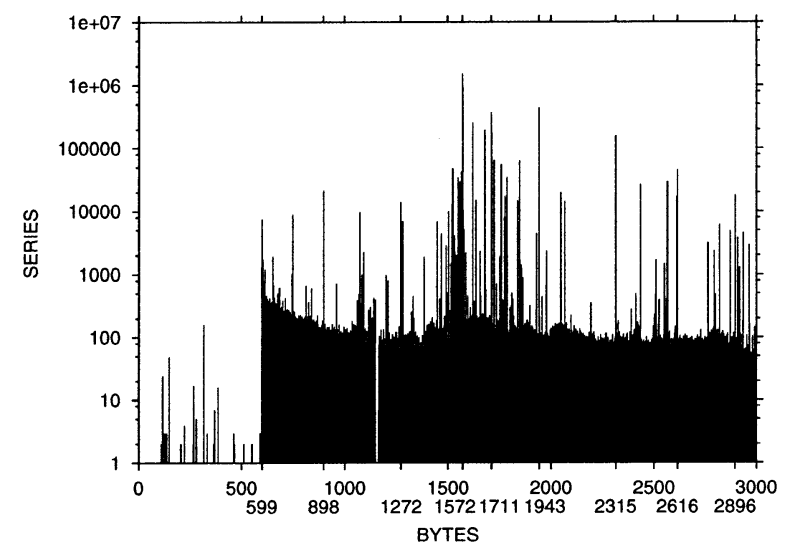

Fig. 12. Enlargement of the 0-3000-byte range of the number of bytes transmitted per correct series for trace AIX-2. Note that this includes the bytes in all of the IP headers for each fragment.

of fragmented IP traffic as observed on all links studied. We analyzed the size (in bytes) of monitored fragment series, the number of fragments in each series, the sizes of the largest and smallest fragments in each series, and the effects of fragments larger than 1500 bytes.

Bytes Per Fragment Series (Fig. 11): The size of the payload carried by each fragment series is highly variable. It has a random component similar to distributions of packet size in general, with a band between 1520 and 1636 bytes per fragment series. Tunneled traffic is a major cause of fragment series in this size range. The source host sends these original datagrams at 1500 bytes - the MTU of Ethernet (and many other link types) — and then they have between 1 and 4 additional IP (or other) headers prepended at the tunnel ingress point. This banding effect and the prevalence of original datagram sizes around 1500 bytes can be seen in Fig. 12, an enlargement of the $0-3000$ byte range of Fig. 11. The most frequently occurring series size across all of the data sets was 1572 bytes. We observe a background, relatively uniform distribution of packet sizes that stretches across series size graphs. In this case, series with total sizes between 597 and around 4000 bytes occurred with a uniform frequency of approximately one hundred series per size. A

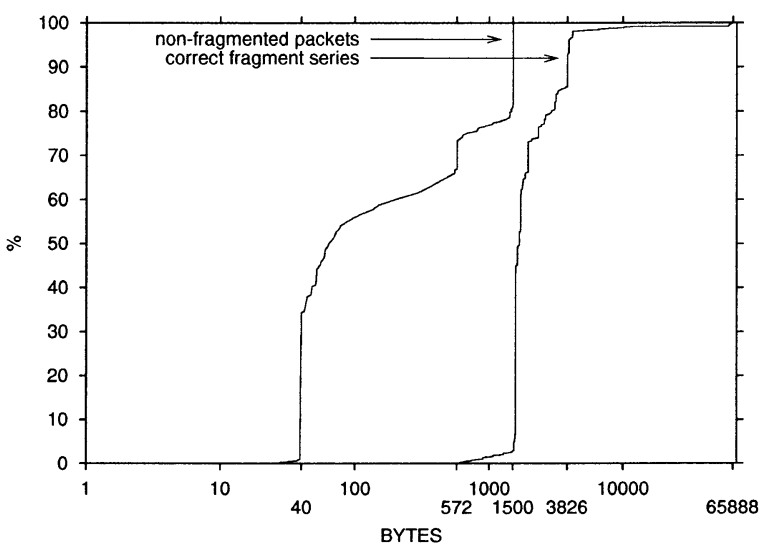

Fig. 13. Cumulative distribution of fragmented and nonfragmented bytes in each IP packet for trace AIX-2. For fragmented traffic, this includes the bytes in all of the IP headers for each series.

background level of approximately 10 series spanned the range from 4000 bytes to 10000 bytes.

Fig. 13 shows the overall packet size distribution for this data set, including both fragmented and nonfragmented traffic. All packet sizes above 30 bytes occur at a frequency in excess of 100000 packets. The most frequently occurring packet size was 40 bytes with 2.69 billion packets, followed by 1500 bytes at 1.49 billion packets and 576 bytes with 514 million packets.

Fig. 12 shows evidence of fragmentation caused by MTU misconfiguration. We monitored a total of 93 series less than 256 bytes. While two of these series appeared to be deliberate optimizations for slow links, the majority appear to be errors. Indeed, the smallest series, at 92 bytes, had only 52 bytes of payload. The overhead for this series, 40 bytes, is nearly as large as the size of the payload. An additional 252 series are considered "poorly configured" because they have series lengths less than 576 bytes. While in a few instances (e.g., routers handling predominantly voice over IP traffic) a low MTU is an optimal configuration, MTUs lower than 576 bytes are generally evidence of mistaken or misguided configuration.

Some end hosts that make modem connections via SLIP set low MTUs for their dial-up link; however, an MTU of 576 is often sufficient to preserve interaction even during large file transfers. The additional overhead incurred should be considered whenever a smaller MTU is chosen: $7 \%$ of 576-byte TCP packets is used by headers necessary for delivery of the packet, while $16 \%$ of 256-byte packets and $31 \%$ of 128 byte packets constitute overhead. To deliver the payload of a 576-byte TCP packet, three 256-byte packets (generating 80 extra bytes) or seven 128-byte packets (generating 240 extra bytes) are necessary.

One phenomenon we often observe in series size histograms is a large original datagram occurring at a frequency disproportionate to its size. These spikes appear to be a transient property of the traffic on each link; they vary in datagram size and magnitude of occurrence over time on the same link and also vary across WAN locations. This data set contains two easily identifiable manifestations of this phenomenon: 14087 fragment series of 60828 bytes and 39114 series of 65888 bytes. Because these large datagrams occur on all links monitored, we will make note 


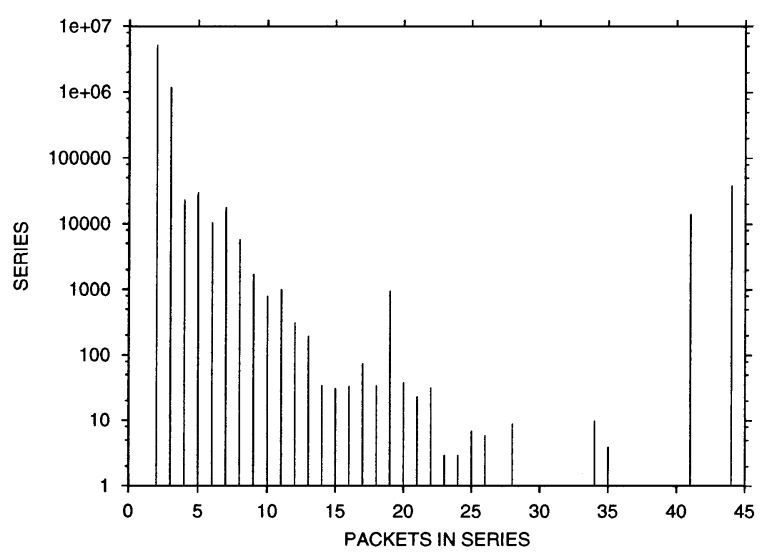

Fig. 14. Number of fragment packets for correct series for trace AIX-2.

of the effects of these occurrences throughout the following sections. Both of these sets of large series are ICMP echo requests and thus may have been "ping of death" attacks ${ }^{3}$ [16].

These fragment series have the following compositions:

Each 60828 -byte fragment series consists of 40 fragments of 1500 bytes followed by 1 fragment of 828 bytes, with original datagram length of 60028 bytes. In this case, 800 bytes of overhead ( $60828-60028)$ were caused by the 40 additional IP headers needed to transmit the series.

Each 65 888-byte fragment series consists of 43 fragments of 1500 bytes followed by 1 fragment of 1388 bytes, with original datagram length of 65028 bytes. In this case, 860 bytes of overhead (65 888-65 028) were the result of the 43 additional IP headers needed to transmit the series.

Fragments Per Fragment Series (Fig. 14): Fragment series are typically two fragments in length. A high number of twofragment series is consistent with a high volume of tunneled fragmented traffic, since this series length accounts for original datagrams that range from just exceeding the MTU of the next link to 40 bytes (for the next packet header) less than double the MTU of the next link:

$$
M T U<\text { datagram } \leq(2 * M T U)-40 .
$$

This spike in two-fragment series in Fig. 14 is generally followed by decreasing numbers of packets with increasing length of the series. We often observe a pairing of even and odd lengths that results in a step-like decrease in the frequency of occurrence of long fragment series. This behavior can be seen in the pairs $(4,5),(6,7),(10,11),(14,15),(21,22),(23,24)$, and $(25,26)$.

We observed an unusually large number of 41 and 44 fragment series at AIX because of the unusual frequency of packets of lengths 60028 and 65028 bytes, respectively. These packets were broken up into 1500-byte fragments with one oddly sized leftover fragment.

Largest Fragment Size Distribution (Fig. 15): The size of the largest fragment found in a fragment series is indicative of the

\footnotetext{
${ }^{3}$ Machines running older versions of many operating systems can be crashed by sending them a ping packet larger than 65535 bytes. Because few protocols allow packets that large, the "ping of death" packet typically arrives as a fragment series, is reassembled by the target machine into the original 65535 -byte packet, and then crashes the target machine.
}

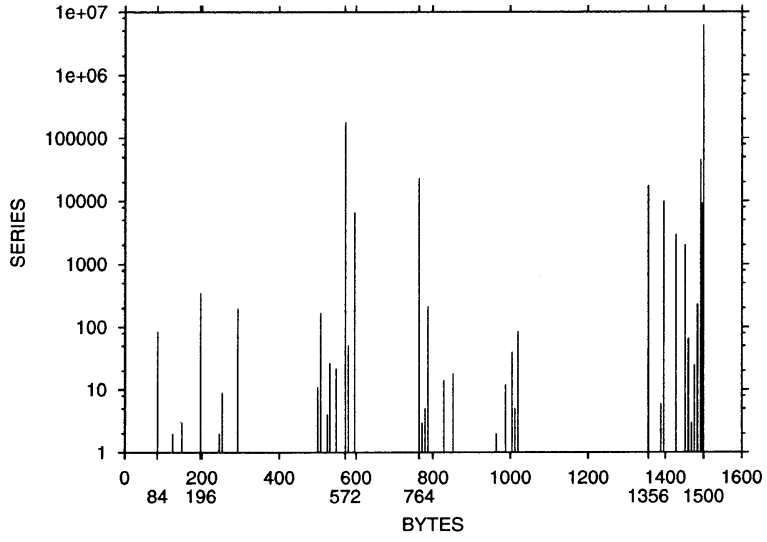

Fig. 15. Largest fragment size for a correct series for trace AIX-2.

TABLE IV

Top Ten Largest Fragments From a Correct Series

\begin{tabular}{l|c}
\hline Fragment Size (bytes) & Series(\%) \\
\hline \hline 1500 & 79. \\
1484 & 18. \\
1492 & 1.0 \\
572 & 0.96 \\
1496 & 0.37 \\
1356 & 0.15 \\
1396 & 0.10 \\
124 & 0.093 \\
764 & 0.081 \\
1452 & 0.055 \\
\hline
\end{tabular}

MTU of the link provoking fragmentation. Typically the first fragment in a fragment series has this maximum size, but this is not universally true. We identified in the AIX and MAE-west data a total of 237263 two-fragment series in which the smallest fragment was received first, with the largest trailing. While only $7.8 \%$ of the total correct fragment series were transmitted in reverse order, we cannot make the assumption that the first fragment of each series is always the largest.

The same misconfigurations that were apparent in the bytes-per-fragment series graph are visible here: it is unlikely that a packet would need to be fragmented to a size less than 576 bytes as it travels toward an exchange point. However, there are no observable artifacts of the 60028 or 65028 original datagram phenomena in this graph. All of those anomalies result in 1500-byte largest fragments, and since 1500 is by far the most common largest fragment size, the anomalies are not visible in the largest fragment size distribution.

Many of the largest fragments occur at sizes easily predicted from the MTUs of common link types. Table IV shows the largest fragment size per series seen across all data sets. The most common largest fragment size by far is 1500 bytes; it is the maximum packet size for Ethernet networks. Ethernet networks using LLC/SNAP, in accordance with RFC 1042 [17], produce 1492-byte IP packets. DEC Gigaswitch traffic results in packets of length 1484 bytes. A widely used PPP MTU is 572 bytes and also results from usage of the default 576-byte transmission size. According to RFC 791 [5] and RFC 879 [18], the largest size packet that a host is required to accept is 576 bytes. Therefore, when Path MTU discovery fails or is not implemented, packets 
are sent at a size less than or equal to 576 bytes. Note that, for IPv6, the minimum MTU of any link must be 1280 bytes [19].

The default packet size of 576 bytes results in fragments of 572 bytes because the length of the payload of each fragment packet except the last must be divisible by eight. This size requirement is based on the design of the IP packet header that specifies that the offset field holds the position of each fragment within the original datagram in eight-byte units [5]. The size of the entire fragment is the sum of the length of the IP header and the payload. Since IP options rarely occur, the IP headers of these fragments are 20 bytes in length [3]. Therefore, the entire packet size for nonlast fragments is $20+N * 8$ for some $N$. The largest valid fragment packet size less than or equal to the default transmission size of 576 bytes is 572 bytes. Such a packet would consist of 20 bytes of IP header and 69 eight-byte units of fragment payload.

Many large fragment sizes evince configuration errors. This is evidence for the utility of Path MTU Discovery, since there is no "safe" transmission size at which a host can send packets to prevent fragmentation without an unacceptable increase in per-packet overhead.

Effects of Fragments Larger Than 1500 Bytes: As we have seen in the previous graphs, the most frequently occurring original datagram sizes shape the characteristics of their resulting fragments. Fragment traffic at MAE-west is unusual in that a common largest fragment size for this link is 4348 bytes, rather than the usual sizes smaller than 1500 bytes. The MAE-west location monitored for this study is an ATM link, and the MTU for IP over ATM is 9180 bytes [20], [21]. Fragments larger than 1500 bytes are more likely to be fragmented again before they reach their destination than smaller fragments.

\section{Fragmented Traffic Protocols and Applications}

This section examines the services, protocols and applications that contribute to fragmented traffic. A comparison of the traffic protocols of fragmented and nonfragmented traffic is shown in Table V.

Services Causing Fragmentation: While many hypothesize that NFS causes all of the fragmented traffic on LAN's and backbone networks, in our data streaming media and tunneled traffic are the dominant cause of IP packet fragmentation.

Streaming media accounts for 53\% of all fragmented traffic. A single application in this group, Microsoft Media Player, is responsible for the $52 \%$ of all fragmented traffic. The use of a protocol that utilizes Path MTU Discovery could significantly reduce the impact of this application on packet fragmentation.

The other major culprit, fragmented tunneled traffic, consists of IP packets sized at the MTU of their local network which were then tunneled, causing the addition of at least one additional IP header. For example, a 1500 byte packet with a 20 byte IP header added as it is tunneled via IPENCAP results in a 1520 byte datagram that exceeds the MTU of the subsequent link, and is fragmented into a 1500 byte first fragment and a 40 byte second fragment. This fragmentation is entirely preventable - an end host that is known to send traffic through an IP tunnel could set the MTU of the interface associated with the tunnel to 1480 bytes,
TABLE V

PROTOCOL BREAKDOWN FOR FRAGMENTED AND NONFRAGMENTED IP TRAFFIC. PERCENTAGES ARE OF TOTAL TRAFFIC

\begin{tabular}{l|r|c|c|c|c}
\hline \multicolumn{2}{|c|}{ Protocol } & \multicolumn{2}{c|}{ Fragmented } & \multicolumn{2}{c}{ Non-Fragmented } \\
\hline Name & Number & Pkts(\%) & Bytes(\%) & Pkts(\%) & Bytes(\%) \\
\hline \hline UDP & 17 & 0.30 & 0.80 & 12. & 3.7 \\
IPENCAP & 4 & 0.061 & 0.11 & 0.12 & 0.039 \\
ESP (IPSEC) & 50 & 0.014 & 0.025 & 0.28 & 0.28 \\
ICMP & 1 & 0.044 & 0.14 & 1.9 & 0.45 \\
TCP & 6 & 0.0076 & 0.018 & 85. & 94. \\
GRE & 47 & 0.0051 & 0.0088 & 0.18 & 0.13 \\
IPIP & 94 & 0.0043 & 0.0074 & 0.033 & 0.021 \\
AH (IPSEC) & 51 & 0.0018 & 0.0032 & 0.053 & 0.042 \\
IGMP & 2 & 0.0015 & 0.0020 & 0.0004 & $<0.0001$ \\
AX.25 & 93 & $<0.0001$ & $<0.0001$ & 0.0045 & 0.0014 \\
\hline
\end{tabular}

rather than 1500 . This would reduce the switching load resulting from the tunneled traffic by $98.7 \%$ - the machine would generate an extra packet for only every seventy-fifth packet sent, rather than requiring a second packet for every original datagram sent from the machine.

Path MTU discovery should allow the end host to discover an MTU that minimizes fragmentation of its tunneled traffic. However, $98.98 \%$ of the fragmented IPENCAP traffic monitored on the link between UCSD and CERFnet in the course of this study consisted of an IP packet with its Don't Fragment bit set, encapsulated by an IP header with no Don't Fragment bit. The end host believes that it is correctly performing Path MTU Discovery, and is oblivious to the fact that packets it sends through the tunnel are fragmented.

To prevent unnecessary fragmentation, implementations of tunneling protocols need to perform Path MTU discovery between the tunnel ingress point and the tunnel egress point and correctly forward ICMP "datagram too big" messages back to source hosts [22]. As the use of tunneling protocols becomes increasingly widespread, increased fragmentation caused by improperly implemented tunneling software may cause performance problems.

Tunneled traffic is not a local area network phenomenon. The combination of IPENCAP, IPIP, GRE, and UDP-L2TP accounts for $22 \%$ of all fragment series-the second largest single cause of fragmentation. None of these protocols currently implement any form of Path MTU discovery. NFS accounts for only $0.1 \%$ of wide-area network fragment series. The most frequently fragmented protocol is IGMP-some $78 \%$ of IGMP packets are fragments. However, since IGMP accounts for only $0.0004 \%$ of all measured packets, this fact is of purely academic import. Table VI contains the protocol breakdown for fragmented traffic, while Table VII displays non-fragmented traffic.

As shown in Table VI, UDP accounts for more fragmented packets than any other protocol- $68.3 \%$ of fragmented traffic. Fragmented ICMP traffic consists primarily $(98.1 \%)$ of echo requests and replies, although a small but significant number of timestamp requests were also monitored. Path MTU Discovery successfully limits the amount of TCP traffic that is fragmented; however, its effects are not quite as ubiquitous as some might claim. More than three million packets over the course of a week, $0.009 \%$ of the total TCP traffic, consisted of fragmented packets. Fragmented TCP traffic does exist on highly aggregated links. 
TABLE VI

PROTOCOL BREAKDOWN FOR FRAGMENTED TRAFFIC. SERIES COLUMN IS FOR CORRECT SERIES ONLY

\begin{tabular}{l||c|c|l}
\hline \multicolumn{1}{c||}{ Protocol } & \multicolumn{3}{c}{ Fragmented } \\
\hline Name & Pkts(\%) & Bytes(\%) & Series(\%) \\
\hline \hline UDP & 68. & 72. & 70. \\
IPENCAP & 14. & 9.7 & 19. \\
ESP (IPSEC) & 3.2 & 2.3 & 4.4 \\
ICMP & 10. & 12. & 2.1 \\
TCP & 1.7 & 1.6 & 2.0 \\
GRE & 1.2 & 0.79 & 1.6 \\
IPIP & 0.97 & 0.67 & 1.0 \\
AH (IPSEC) & 0.40 & 0.29 & 0.54 \\
IGMP & 0.33 & 0.18 & 0.024 \\
AX.25 & 0.0009 & 0.0001 & 0.0011 \\
\hline
\end{tabular}

TABLE VII

PROTOCOL BREAKDOWN FOR NONFRAGMENTED TRAFFIC

\begin{tabular}{l||c|c}
\multicolumn{1}{c||}{ Protocol } & \multicolumn{2}{c}{ Non-Fragmented } \\
\hline Name & Pkts(\%) & Bytes(\%) \\
\hline \hline UDP & 12. & 3.8 \\
IPENCAP & 0.12 & 0.040 \\
ESP (IPSEC) & 0.28 & 0.28 \\
ICMP & 1.9 & 0.45 \\
TCP & 85. & 95. \\
GRE & 0.18 & 0.13 \\
IPIP & 0.033 & 0.021 \\
AH (IPSEC) & 0.053 & 0.043 \\
IGMP & 0.0004 & $<0.0001$ \\
AX.25 & 0.0045 & 0.0015 \\
\hline
\end{tabular}

TABLE VIII

TOP TCP APPLICATIONS FROM CORRECT FRAGMENT SERIES

\begin{tabular}{l|c}
\hline TCP Application & Series $(\%)$ \\
\hline \hline SMTP & 51. \\
FTP_DATA & 36. \\
HTTP & 4.8 \\
NAPSTER_DATA & 4.7 \\
Unclassified TCP & 2.8 \\
GNUTELLA & 0.60 \\
X11 & 0.066 \\
BGP & 0.019 \\
SSH & 0.017 \\
KERBEROS & 0.0079 \\
\hline
\end{tabular}

TCP Applications (Table VIII): Of the fragmented TCP series, $50.8 \%$ are composed of SMTP packets. Two peer-to-peer file-sharing applications, Napster and Gnutella, account for a total of 5.3\% of fragmented TCP traffic, despite being a larger portion of nonfragmented traffic. However, we only identify Napster and Gnutella traffic on the most commonly used ports. Because Gnutella servers and, to a lesser extent, Napster servers often use alternate ports (typically to circumvent blocks intended to impede use of these applications), we underestimate, perhaps significantly, the prevalence of both fragmented and nonfragmented peer-to-peer file-sharing application use.

ICMP Applications (Table IX): The majority (98\%) of ICMP traffic observed was associated with echo request and reply. Thirteen percent of ICMP echo requests were series of 65888 bytes (previously discussed in Section IV-C). The $1.8 \%$ of fragmented ICMP traffic which was a timestamp request appears to have been either a misconfiguration or a
TABLE IX

TOP ICMP APPLICATIONS From CORRECT FraGMENT SERIES

\begin{tabular}{l|c}
\hline \multicolumn{1}{c|}{ ICMP Application } & Series(\%) \\
\hline \hline Echo Request & 61. \\
Echo Reply & 37. \\
Timestamp Request & 1.8 \\
Port Unreachable & 0.039 \\
TTL Expired - Reassembly & 0.0041 \\
Host Unreachable & 0.0028 \\
Frag Needed But "DF" Set & 0.0020 \\
Type 69, Code 0 & 0.0003 \\
TTL Expired - Transit & 0.0001 \\
\hline
\end{tabular}

TABLE $X$

TOP TEN UDP APPLICATIONS USING EPHEMERAL PORTS

\begin{tabular}{l|r|c}
\hline UDP Application & Series(\#) & \multicolumn{1}{c}{ Series(\%) } \\
\hline \hline MS_MEDIA & $6,461,564$ & 82. \\
UNKNOWN & $1,370,495$ & 17. \\
ASHERONS & 12,132 & 0.15 \\
TALK & 12,057 & 0.15 \\
ISAKMP & 7,543 & 0.095 \\
BIFF & 5,871 & 0.074 \\
DAYTIME & 4,067 & 0.051 \\
POINTCAST & 4,038 & 0.051 \\
ICU_II & 3,030 & 0.038 \\
NFS & 3,027 & 0.038 \\
\hline \multicolumn{2}{|c}{}
\end{tabular}

TABLE XI

TOP TEN TCP AND UDP APPLICATIONS AND APPLICATION GROUPS OVERALL (INCLUDING APPLICATIONS ON EPHEMERAL PORTS IDENTIFIED VIA CONTROL STREAMS)

\begin{tabular}{l|c}
\hline Application & Series(\%) \\
\hline \hline MS_MEDIA & 78. \\
UNKNOWN & 16. \\
QUAKE & 2.4 \\
L2TP & 1.7 \\
DOOM & 0.86 \\
REALAUDIO_UDP & 0.19 \\
ASHERONS & 0.14 \\
TALK & 0.14 \\
AOL & 0.11 \\
ISAKMP & 0.090 \\
\hline
\end{tabular}

\begin{tabular}{l|c}
\hline Application Group & Series(\%) \\
\hline \hline Streaming & 78. \\
UNKNOWN & 16. \\
Games & 3.4 \\
Tunneling & 1.9 \\
Other & 0.28 \\
Network_Infrastructure & 0.25 \\
File_Systems & 0.14 \\
Encryption & 0.12 \\
Conferencing & 0.10 \\
WwW & 0.076 \\
\hline
\end{tabular}

denial-of-service attack. An ICMP timestamp request packet is normally 20 bytes plus the IP header and thus should never be larger than 80 bytes. The observed fragmented ICMP timestamp traffic predominantly used payloads of 5013 bytes with less than $1 \%$ using 4013 and 7513 bytes. In some instances multiple source hosts were sending the same type of 5013-byte ICMP timestamp messages to the same destination, suggesting a denial-of-service attack.

UDP Applications (Tables $X$ and XI): Ninety-eight percent of all fragmented UDP traffic occurs on dynamically allocated ports. To identify the applications associated with traffic on these ephemeral ports, we must match these ambiguous fragments with control traffic on known ports. However, because our initial study did not include a packet-level trace of unfragmented traffic, we collected $16 \mathrm{~h}$ (including business hours) of TCP, UDP, and ICMP flow data from our MAEWEST, UCSD, and SDNAP taps on February 26, 2002. The distribution of applications in this follow-up study was similar to that of the traces used in the earlier study. Specifically, Unclassified UDP 
traffic accounted for $93.11 \%$ of all observed UDP traffic, a reduction of 5\% from the previous study. The utilization of a few applications, including the games Quake, Doom, and Halflife, Microsoft's Media Player, and AOL increased, otherwise the overall distribution of applications remained the same.

At each location, we built a tuple table based upon the source IP address, destination IP address, protocol, and ports of each measured flow. We identified UDP flows with fragmented packets and extracted all other flows involving the same source and destination IP that occurred within $120 \mathrm{~s}$ of the fragmented flow. We explored the effect of the timeout window size on the number of matched and unmatched fragment series. We chose $120 \mathrm{~s}$ as the window size for this study because it minimizes unmatched fragment series while minimizing multiple matches for each fragment series.

When we generated multiple matches for a single unknown UDP series despite tuning the window size, we attempted to determine which of the matches was the most likely control stream for the fragments. We removed duplicate matches and eliminated a few common applications (traceroute, http, netbios) that are never control streams for UDP traffic on ephemeral ports. If multiple matches remained, we chose the match that occurred most commonly on its own. For example, if we narrowed the choices to Microsoft's RealMedia Player (81\%) and Squid $(<0.1 \%)$, we'd assign the unidentified fragments to Microsoft's RealMedia Player. Varying our method of resolving multiple matches has no significant effect on the final results.

As seen in Table XI, the vast majority of fragmented UDP traffic appears to be caused by Microsoft's Windows Media Player. Other culprits include the games Quake, Doom, and Asheron's Call, and L2TP, a tunneling protocol.

We also categorized applications identified via well-known port numbers into 13 groups ${ }^{4}$ based on similarities among application functions. The top ten application groups contributing to fragmented traffic, as measured in February 2002, are shown in Table XI. While Streaming applications are the primary contributors of fragmented traffic, the effects of tunneled traffic are underrepresented in this table because several tunneling protocols (IPSEC, GRE) are not identifiable via port numbers.

IPv6 and Packet Fragmentation: The next version of the IP protocol, IPv6, eliminates the IP packet fragmentation mechanism in routers [19].

IPv6 also requires a checksum in the UDP header of all UDP packets. The UDP checksum field does appear in the IPv4 UDP header, but its use is optional. One proposed mechanism for bridging IPv4 and IPv6 networks is that UDP packets lacking checksums will have checksums computed and applied before they are transmitted onto IPv6 networks. This process of checksum computation is difficult for fragmented traffic since all of the fragments of the original datagram must be reassembled before a checksum can be computed. If all of the fragments do not share the same egress point from the IPv4 network, checksum computation is impossible. However, we are aware of no available data on the prevalence of IPv4 UDP fragments without UDP checksums. In our data, we observe

\footnotetext{
${ }^{4}$ Application Groups: Conferencing, Encryption, File Systems, File Transfer, Games, Login, Mail/News, Network Infrastructure, Other, P2P, Streaming, Tunneling, and WWW.
}

that only $0.42 \%$ of all UDP fragments lacked a UDP checksum. However, $25.5 \%$ of all hosts sending fragmented traffic sent UDP packets without checksums. $82.3 \%$ of all hosts that sent UDP packets without a checksum also sent UDP packets with checksums. This result is consistent with application-specific checksum incorporation, rather than host-specific behavior, which complicates a user-transparent IPv4 to IPv6 transition.

\section{CONCLUSION}

Many assertions about the nature and extent of fragmented traffic are based in folklore rather than on measurement and analysis. Common beliefs include: fragmented traffic is decreasing in prevalence or nonexistent, fragmented traffic exists only on LANs (due to NFS) and not on backbone links, misconfiguration causes most fragmentation, and only UDP traffic is fragmented.

While the majority of fragmented traffic is UDP (68\% by packets and $72 \%$ by bytes), ICMP, IPSEC, TCP, and tunneled traffic are commonly fragmented as well. Microsoft's Media Player is the single largest source of fragment series, accounting for $52 \%$ seen in this study. Tunneled traffic is a major cause of fragmented traffic, and accounts for at least $16 \%$ of fragmented series.

NFS accounts for only $0.1 \%$ of fragment series observed. We were unable to classify the applications associated with a small percentage of UDP traffic because of the use of ephemeral ports and dynamically exchanged ports. The classifiable UDP traffic was comprised primarily of tunneled, streaming media and game traffic.

Fragmented traffic does occur regularly at highly aggregated exchange points as well as on access links. Although fragmented traffic is a small percentage of traffic overall, its prevalence is highly variable; fragmented traffic accounted for $8 \%$ of all packets during hour-long periods on some links. The bursty nature of fragmented traffic makes it difficult to measure accurately with short samples of network traffic.

Fragmented traffic is detrimental to WAN performance. Fragmented traffic causes increased load on routers, through both the division of the original packet and the increased number of packets handled by all subsequent routers. The traffic also causes increased load on links due to the overhead of an extra IP header for each fragment. Additionally, because all of the fragments are necessary to reassemble the original packet, the probability of successfully delivering a fragmented packet exponentially decreases as a function of the number of fragments, in contrast to the normal packet loss rate. This partial packet loss may further increase link and router loading as higher layers must retransmit packets.

With the advent of IPv6, all packets that are currently fragmented in the network will be dropped by routers, with a "Packet Too Big" ICMP message returned to the source host [23]. The proposed mechanism for transition between IPv4 and IPv6 networks requires checksums for all fragmented UDP traffic, yet $26 \%$ lacks a UDP checksum. Understanding the actual prevalence and causes of fragmented traffic is critical to the success of currently proposed protocols and security efforts. 


\section{ACKNOWLEDGMENT}

The authors would like to thank R. Koga for writing the program used to collect data for the first part of this study and K. Keys for suggestions and help using CoralReef. This paper would not have been possible without the assistance of S. Feldman, B. Cates, and J. Pucik with the MAE-west and AIX passive monitors. The authors are especially grateful for the assistance of D. Plummer, D. Meyer, B. Fenner, E. Nemeth, C. Partridge, K. Keys, G. Voelker, and the SIGCOMM Internet Measurement Workshop reviewers and participants, and the ACM Transactions on Networking reviewers for useful suggestions for this paper. As always, the support of the CAIDA staff was invaluable.

\section{REFERENCES}

[1] C. A. Kent and J. C. Mogul, "Fragmentation considered harmful,", WRL Tech. Rep.87/3, Dec. 1987

[2] G. P. Chandranmenon and G. Varghese, "Reconsidering fragmentation and reassembly," in Proc. PODC: 17th ACM SIGACT-SIGOPS Symp. Principles of Distributed Computing, 1998.

[3] S. McCreary and K. Claffy, "Trends in wide area IP traffic patterns: A view from Ames Internet Exchange," in Proc. ITC Specialist Seminar on IP Traffic Modeling, Measurement and Management, Sept. 2000.

[4] J. C. Mogul and S. E. Deering, RFC 1191: Path MTU discovery, Nov. 1990.

[5] J. Postel, "RFC 791: Internet Protocol,", Sept. 1981

[6] CERT Advisory CA-1997-28 IP Denial-of-Service Attacks [Online]. Available: http://www.cert.org/advisories/CA-1997-28.html

[7] J. Anderson. (2001, Mar.) An analysis of fragmentation attacks. [Online]. Available: http://www.sans.org/infosecFAQ/threats/frag_attacks.htm

[8] Applied Telecom (apptel). Product overview: Pci optical interface network transceiver. [Online]. Available: http://www.apptel.com/ pdf/ov_point.pdf

[9] S. McCanne, C. Leres, and V. Jacobson, "libpcap," Lawrence Berkeley Laboratory, Berkeley, CA, available via anonymous ftp to ftp.ee.lbl.gov.

[10] Marconi Corporation. Marconi-ForeRunner 200e nics. [Online]. Available: http://www.marconi.com/html/solutions/forerunner200enics.htm

[11] Waikato Applied Network Dynamics Group. The DAG project. [Online]. Available: http://dag.cs.waikato.ac.nz/

[12] K. Keys, D. Moore, R. Koga, E. Lagache, M. Tesch, and K. Claffy, "The architecture of CoralReef: An Internet traffic monitoring software suite," in PAM2001-A Workshop on Passive and Active Measurements: CAIDA, RIPE NCC, Apr. 2001, [Online]. Available: http://www.caida.org/tools/measurement/coralreef/.

[13] IANA port assignments [Online]. Available: ftp://ftp.isi.edu/in-notes/ iana/assignments/port-numbers

[14] D. Moore, G. M. Voelker, and S. Savage, "Inferring Internet denial-ofservice activity," presented at the Usenix Security Symp., 2001.

[15] Y. Zhang, V. Paxson, and S. Shenker, "The stationarity of Internet path properties: Routing, loss, and throughput," ACIRI Tech. Rep., May 2000.

[16] M. Kenney. Ping of death. [Online]. Available: http://www.insecure.org/sploits/ping-o-death.html
[17] J. Postel and J. K. Reynolds, "RFC 1042: Standard for the transmission of IP datagrams over IEEE 802 networks,", Feb. 1988.

[18] J. Postel, "RFC 879: The TCP maximum segment size and related topics,", Nov. 1983.

[19] S. Deering and R. Hinden, "RFC 2460: Internet Protocol, Version 6 (IPv6) specification,", Dec. 1998.

[20] R. Atkinson, "RFC 1626: Default IP MTU for use over ATM AAL5,", May 1994.

[21] M. Laubach and J. Halpern, "RFC 2225: Classical IP and ARP over ATM,", Apr. 1998.

[22] C. Perkins, "RFC 2003: IP encapsulation within IP,", Oct. 1996.

[23] A. Conta and S. Deering, "RFC 2463: Internet Control Message Protocol (ICMPv6) for the Internet Protocol Version 6 (IPv6) specification,”, Dec. 1998.

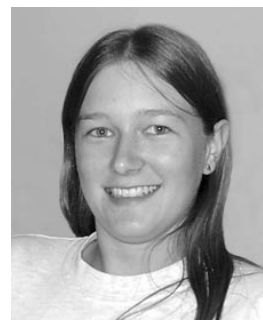

Colleen Shannon is a Staff Researcher at the Cooperative Associate for Internet Data Analysis (CAIDA), University of California at San Diego, La Jolla. Her research interests include traffic measurement, characterization, and modeling, particularly with regard to network security and network application identification.

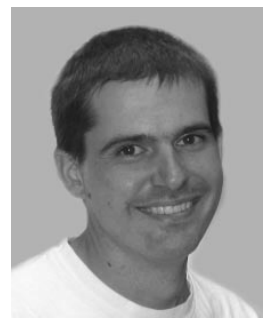

David Moore is a Principal Investigator and Assistant Director of the Cooperative Association for Internet Data Analysis (CAIDA), University of California at San Diego, La Jolla. His research interests are high-speed network monitoring, denial-of-service attacks and infrastructure security, and Internet traffic characterization. In addition to network analysis, he has led several tool development projects ranging from data collection to geographic mapping.

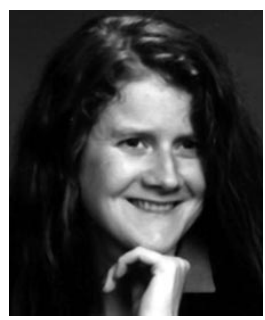

K. C. Claffy is a Principal Investigator for the Cooperative Association for Internet Data Analysis (CAIDA), University of California at San Diego, La Jolla. Her research interests include: data collection, analysis, and visualization of Internet workload, performance, topology, and routing behavior; measurement in the face of ISP commercialization/competition; impact of high-demand (e.g., multimedia) applications on the integrity of the infrastructure; the changing financial structure of the Internet; and equity among users. 